\title{
Efficiently Decodable and Searchable Natural Language Adaptive Compression *
}

\author{
Nieves R. Brisaboa \\ Fac. de Informática, \\ Univ. da Coruña, \\ A Coruña, Spain. \\ brisaboa@udc.es
}

\author{
Antonio Fariña \\ Fac. de Informática, \\ Univ. da Coruña, \\ A Coruña, Spain. \\ fari@udc.es
}

\author{
Gonzalo Navarro \\ Dept. of Computer Science, \\ Univ. of Chile, \\ Santiago, Chile. \\ gnavarro@dcc.uchile.cl
}

\author{
José R. Paramá \\ Fac. de Informática, \\ Univ. da Coruña, \\ A Coruña, Spain. \\ parama@udc.es
}

\begin{abstract}
We address the problem of adaptive compression of natural language text, focusing on the case where low bandwidth is available and the receiver has little processing power, as in mobile applications. Our technique achieves compression ratios around $32 \%$ and requires very little effort from the receiver. This tradeoff, not previously achieved with alternative techniques, is obtained by breaking the usual symmetry between sender and receiver present in statistical adaptive compression. Moreover, we show that our technique can be adapted to avoid decompression at all in cases where the receiver only wants to detect the presence of some keywords in the document, which is useful in scenarios such as selective dissemination of information, news clipping, alert systems, text categorization, and clustering. We show that, thanks to the same asymmetry, the receiver can search the compressed text much faster than the plain text. This was previously achieved only in semistatic compression scenarios.
\end{abstract}

\section{INTRODUCTION}

Text compression [2] permits representing a document using less space. This is useful not only to save disk space, but more importantly, to save disk transfer and network transmission time. In recent years, compression techniques especially designed for natural language texts have not only proven extremely effective (with compression ratios around 25\%-30\%), but also permitted searching the compressed text much faster (up to 8 times) than the original text $[20,9,10]$. The integration of compression and indexing techniques [21, $17,25]$ opened the door to compressed text databases, where texts and indexes are manipulated directly in compressed form, and both time and space are simultaneously saved.

\footnotetext{
*Partially supported by CYTED VII.19 RIBIDI Project. Also funded in part (for the Spanish group) by MCyT (PGE and FEDER) grant(TIC2003-06593) and (for the third author) by Millennium Nucleus Center for Web Research, Grant (P01-029-F), Mideplan, Chile.
}

Permission to make digital or hard copies of all or part of this work for personal or classroom use is granted without fee provided that copies are not made or distributed for profit or commercial advantage and that copies bear this notice and the full citation on the first page. To copy otherwise, to republish, to post on servers or to redistribute to lists, requires prior specific permission and/or a fee.

Copyright 200X ACM X-XXXXX-XX-X/XX/XX $\ldots \$ 5.00$.
The key to the success of natural language text compression is the use of a word-based model [15], so that the text is regarded as a sequence of words. This poses the overhead of managing a large source alphabet, but in large text collections the vocabulary size is relatively insignificant because of Heaps Law [12]. Since the distribution of words is rather biased, following a Zipf Law $[22,1]$, the sequence of words is highly compressible with a zero-order encoder such as Huffman code [14]. In order to be searchable, semistatic models have been used in compressed text databases, to ensure that the codeword assigned to a word does not change across the text. Thus, a pattern can be compressed and directly searched for in the compressed text without decompressing it. This is also essential to permit local decompression of text passages in order to present them to the final users. Different searchable, word-based, semistatic statistical compressors with different merits have been used, such as bit-oriented Huffman [20], byte-oriented Huffman and variants [10], and the more recent End-Tagged Dense Codes and $(s, c)$-Dense Codes $[6,4]$.

As explained, documents are decompressed only in order to present them. A problem not addressed with the current schemes is how to transmit individual documents in compressed form. This is very interesting when the text server must transfer the documents through a low-bandwidth network. Note that it is not feasible to directly transfer the document as it is compressed in the database, because the receiver does not know the semistatic model used by the sender (which includes the vocabulary of the whole collection), and the overhead of transmitting it would be prohibitive, as the collection vocabulary is large when compared to the size of individual documents.

Adaptive or dynamic,compression methods do not need to transmit the model because the receiver can learn it as it receives the compressed text. They have the additional advantage over semistatic methods that the sender does not need to perform a first pass over the text to build the model, but it can start the compression and transmission immediately, while the receiver can start reception and decompression simultaneously. A way to transmit individual documents in a compressed text database scenario is that the server uncompresses them and then recompresses them with an adaptive method.

Note that transmitting an individual document with an adaptive scheme might not be very effective because there is not much time to converge to a good model. This is especially valid in word-based models because even the local 
vocabulary of the document (which is transmitted as new symbols as they appear) is relatively large for small documents, again by Heaps Law [12]. Yet, this works well when the client establishes a longer session with the text server and several documents are transmitted along time. This can be precisely the case of a user session in a compressed text database, when browsing a digital library or a Web site, or in a chat or email service.

There are some scenarios where the receiver can be interested not in uncompressing the arriving text, but just in searching it for some specific words, for classification or retrieval purposes. This has applications in selective dissemination of information, news clipping, altert systems and others. In all those cases, documents gathered from different sources are received and pointed out to users when keywords are found that denote topics of interest. Another application is text categorization and clustering, where documents gathered from text servers are classified according to the presence of certain keywords, without need to ever having the text at the categorization machine. For example, a language classification system might look for a small set of common words of each language and use it to classify the incoming compressed text, forwarding it to a specific directory or computer depending of its language, to be indexed, stored, or even automatically translated.

Therefore it would be useful to have a dynamic compression method with direct search capabilities, that is, permitting direct search of the compressed text without decompressing it. However, direct search of text compressed with an adaptive technique is far more difficult than with a semistatic technique, as a given pattern looks different throughout the compressed document. Although there exist direct search techniques for adaptive compression that are more efficient than uncompressing and searching [19], semistatic compression permits much faster searching, faster than just searching the uncompressed text (without counting the time to uncompress) [10].

The best known adaptive compression methods are the Ziv-Lempel family [23, 24] and dynamic arithmetic coding [16]. The former obtains reasonable but not spectacular compression ratios (around 40\%). The latter compresses much better (around 25\%), but it requires significant computational effort both from the sender and from the receiver. This is especially unfortunate in cases where the receiver has limited computational power, such as in mobile applications. This symmetry in the sender and receiver efforts is at the essence of statistical adaptive compression, as both have to update their models in synchronization. It is also central to the difficulty of direct searching text compressed with adaptive methods.

In $[5,11]$, adaptive versions of both word-based byteoriented Huffman and End-Tagged Dense Code (ETDC) were presented. The latter turns out to be especially interesting, achieving around 30\% compression ratio and a compression speed close to the fastest Ziv-Lempel compressors (which achieve only $40 \%$ compression ratio) and 2.5 times faster than word-based arithmetic coding. Decompression is 3.5 times faster than with word-based arithmetic coding, but still twice as slow as the Ziv-Lempel compressors.

In this paper we improve the existing results on wordbased adaptive compression, focusing on reducing the effort of the receiver in order to either uncompress or search the compressed text. We present a variant of the previous Dy- namic End Tagged Dense Code (DETDC), that we call $\boldsymbol{D} \boldsymbol{y}$ namic Lightweight End Tagged Dense Code (DLETDC). DLETDC has almost exactly the same compression ratio of ETDC and DETDC, but it requires much less processing effort from the receiver than DETDC. As a result, decompression time is now similar to that of Ziv-Lempel methods in short files and better in longer files. The key idea is to relief the receiver from maintaining the model as it receives the text, but letting the sender inform of the changes when necessary. For this to be useful, we designed DLETDC so as to maintain its compression ratio while minimizing the required updates to the model. This breaks the usual symmetric model of statistical adaptive compression.

Our second contribution focuses on the case where the receiver does not need to recover the original text, but just to detect the presence of some keywords in it. We show how DLETDC compressed text can be searched without decompressing it. The search algorithm is even lighter than the uncompression algorithm. It needs very little memory and can perform efficient Boyer-Moore-type searching on the compressed text. We show that searching the compressed text for a set of keywords is much faster (up to 5 times) over our compressed text than over the uncompressed text.

This breaks another long-standing assumption that states that only semistatic models permit efficient Boyer-Moore searching on the compressed text. In particular, this is the first adaptive compression scheme that permits searching the compressed text faster than the uncompressed text.

\section{RELATED WORK}

\subsection{Word-based Huffman codes}

Huffman code is the optimal (shortest total length) statistical prefix code for a given text. However traditional implementations of the Huffman code are character-based, (they use characters as source symbols) and therefore they obtain poor (around 65\%) compression ratios. The brilliant wordbased approach, proposed by Moffat [15], yields much better compression ratios, close to $25 \%$, using a binary target alphabet, that is, each word in the source text is encoded as a sequence of bits. In [10] two byte oriented word-based Huffman codes were proposed, called Plain Huffman and Tagged Huffman respectively.

Plain Huffman does not modify the basic Huffman code except by using bytes as the symbols of the target alphabet. It obtains compression ratios close to $30 \%$ on natural language text. The loss in compression is due to the use of bytes instead of bits as in [15]. In exchange, decompression and searching are much faster with Plain Huffman code because no bit manipulations are necessary.

In Tagged Huffman [10], the first bit of each byte is reserved to flag whether the byte is the first of its codeword. Hence, only 7 bits of each byte are used for the Huffman code. Note that the use of a Huffman code over the remaining 7 bits is mandatory, as the flag is not useful by itself to make the code a prefix code. While searching Plain Huffman compressed text requires inspecting all its bytes from the beginning, the tag bit in Tagged Huffman permits BoyerMoore-type searching (that is, skipping bytes) [3] by simply compressing the pattern and then running the string matching algorithm. On Plain Huffman this does not work, as the pattern could occur in the text not aligned to any codeword [10]. The problem is that the concatenation of two code- 
words may contain the codeword of another source symbol. This cannot happen in Tagged Huffman code thanks to the tag bit that distinguishes initial codeword bytes.

On the other hand, Tagged Huffman code pays a price in terms of compression performance of approximately $11 \%$, as it stores full bytes but uses only 7 bits for coding.

\subsection{End Tagged Dense Codes}

End-Tagged Dense Code (ETDC) [6] is obtained by a seemingly dull change to the Tagged Huffman Code [10]. Instead of using a flag bit to signal the beginning of a codeword, the end of a codeword is signaled. That is, the highest bit of any codeword byte is 0 except for the last byte, where it is 1 .

This change has surprising consequences. Now the flag bit is enough to ensure that the code is a prefix code regardless of the contents of the other 7 bits of each byte. To see this, consider two codewords $X$ and $Y$, being $X$ shorter than $Y$ $(|X|<|Y|)$. $X$ cannot be a prefix of $Y$ because the last byte of $X$ has its flag bit in 1, while the $|X|$-th byte of $Y$ has its flag bit in 0 . Thanks to this change, there is no need at all to use Huffman coding in order to maintain a prefix code. Therefore, all possible combinations of bits can be used over the remaining 7 bits of each byte, producing a dense encoding. This yields a better compression ratio than Tagged Huffman while keeping all its good searching and decompression capabilities. On the other hand, ETDC is easier to build and faster in both compression and decompression.

In general, ETDC can be defined over symbols of $b$ bits, although in this paper we focus on the byte-oriented version where $b=8$. ETDC is formally defined as follows.

Definition 1. Given source symbols with decreasing probabilities $\left\{p_{i}\right\}_{0 \leq i<n}$ the corresponding codeword using the End-Tagged Dense Code is formed by a sequence of symbols of $b$ bits, all of them representing digits in base $2^{b-1}$ (that is, from 0 to $2^{b-1}-1$ ), except the last one which has a value between $2^{b-1}$ and $2^{b}-1$, and the assignment is done in a sequential fashion.

That is, the first word is encoded as 10000000 , the second as 10000001 , until the $128^{t h}$ as 11111111 . The $129^{\text {th }}$ word is coded as 00000000:10000000, $130^{\text {th }}$ as 00000000:10000001 and so on until the $\left(128^{2}+128\right)^{t h}$ word $\underline{0} 1111111: 11111111$. Note that the code depends on the rank of the words, not on their actual frequency. As a result, only the sorted vocabulary must be stored with the compressed text for the decompressor to rebuild the model. Therefore, the vocabulary will be slightly smaller than in the case of Huffman codes, where some information about the shape of the Huffman tree must be stored (even when a canonical Huffman tree is used).

It is clear that the number of words encoded with 1,2 , 3 etc, bytes is fixed (specifically $128,128^{2}, 128^{3}$ and so on) and does not depend on the word frequency distribution. Generalizing, being $k$ the number of bytes in each codeword $(k \geq 1)$ then words at positions:

$$
2^{b-1} \frac{2^{(b-1) k-1}-1}{2^{b-1}-1} \leq i<2^{b-1} \frac{2^{(b-1) k}-1}{2^{b-1}-1}
$$

will be encoded with $k$ bytes. These clear limits mark the change points in the codeword lengths and will be relevant in the adaptive version of ETDC that we present in this paper.
As it can be seen, the computation of codes is extremely simple: It is only necessary to sort the source symbols by decreasing frequency and then sequentially assign the codewords. But not only the sequential procedure is available to assign codewords to the words. There are simple encode and decode procedures that can be efficiently implemented, because the codeword corresponding to symbol $i$ is obtained as the number $x$ written in base $2^{b-1}$, where $x=i-\frac{2^{(b-1) k}-2^{b-1}}{2^{b-1}-1}$, and adding $2^{b-1}$ to the last digit.

Function encode obtains the codeword $C_{i}=\operatorname{encode}(i)$ for a word at the $i$-th position in the ranked vocabulary.Function decode gets the position $i=\operatorname{decode}\left(C_{i}\right)$ in the rank for a codeword $C_{i}$. Both functions take just $O(l)$ time, where $l=O(\log (i) / b)$ is the length in digits of codeword $C_{i}$. Those functions are very efficient, because they can be implemented through just bit shifts and masking.

End-Tagged Dense Code is simpler, faster, and compresses $7 \%$ better than Tagged Huffman codes. In fact ETDC only produces an overhead of about $2 \%$ over Plain Huffman. On the other hand, since the last bytes of codewords are distinguished, ETDC has all the search capabilities of Tagged Huffman code. Empirical results comparing ETDC against Plain and Tagged Huffman can be found in [6].

In [4] a generalization of ETDC, called $(s, c)$-Dense Code $((\mathrm{s}, \mathrm{c})-\mathrm{DC})$ was presented. In $(\mathrm{s}, \mathrm{c})-\mathrm{DC}$ a dense codification is also used, but instead of using 128 values (from 0 to 127) as non final bytes of codewords (continuers) and other 128 values (from 128 to 255) as final bytes of codewords (stoppers), a different number of stoppers $(s)$ and continuers $(c)$ are used $\left(s+c=2^{b}\right)$ in order to obtain an optimal compression. Of course, the $s$ and $c$ values depend on the model and are computed during the first pass over the text.

\subsection{Dynamic End-Tagged Dense Codes}

Dynamic techniques start compressing and transmitting the text as soon as the first source symbol is processed. In [5] a dynamic version of End-Tagged Dense Code (DETDC) was presented. As any one-pass technique, DETDC collects word frequencies as the text is read and, consequently, the model is updated as compression progresses. Both sender (compressor) and receiver (decompressor) increment the frequency of each new input word, and maintain the vocabulary ordered by frecuency. Therefore, the sender does not transmit the model, since the receiver can figure it out by itself from the received codewords. The sender informs the receiver of new source symbols appearing in the text, using a special codeword $C_{n e w-S y m b o l}$. The sender transmits $C_{n e w-S y m b o l}$ followed by the source word in ASCII. The receiver inserts it in its vocabulary setting its frequency to one. In DETDC, $C_{n e w-S y m b o l}$ was always the first unused codeword, that is, the codeword that follows that of the last word in the vocabulary. When a word arrives, and it is already in the vocabulary, the sender transmits its codeword, increments its frequency and reorders the vocabulary if necessary. When the receiver gets a codeword other than $C_{n e w-S y m b o l}$, it decodes the codeword so as to obtain the corresponding vocabulary position, recovers the codeword and increments its frequency. Finally it also reorders the vocabulary if necessary.

In DETDC the encode and decode procedures permit to efficiently produce a codeword from the word rank in the compressor, and to efficiently obtain the vocabulary word position, from an arriving codeword, in the receiver. Both 
the sender and the receiver are in charge of maintaining the sorted vocabulary, carrying out two symmetric processes. That is, a word position in the ranked vocabulary is the only necessary data to encode a word, because the mathematical correspondence rank-codeword explained in section 2.2 is used to compute the words-codewords mapping. Details can be found in [5].

\section{DYNAMIC LIGHTWEIGHT END TAGGED DENSE CODE}

Dynamic Lightweight End Tagged Dense Code (DLETDC) is the new dynamic compression technique we introduce in this paper. It is based on DETDC, but it avoids the overhead of keeping the model up to date in the side of the receiver. This makes it extremely convenient in scenarios where the bandwidth is low and the receiver has little processing power, such as in mobile applications. The price is a very slight increase in the processing cost of the sender and in the compression ratio.

In DLETDC, only the sender keeps the frequency of each symbol and maintains the vocabulary sorted by frequency. The receiver, instead, only stores an array of words indexed by their codewords, with no frequency information. When a codeword arrives, the receiver decodes it using the standard decode procedure and obtains the corresponding word position. The receiver does not update the model at all. Therefore, the sender should inform the receiver of any change in the codewords-words mapping. Note that changes in the codeword assignments upon frequency changes are necessary to maintain good compression ratios. However, the number of exchanges in the vocabulary is large enough to affect the compression ratio if all of them have to be informed to the receiver, where they also require some effort to be processed. Hence, we seek at minimizing the number of exchanges without affecting the original compression ratio.

Our basic idea is that only when the increment in frequency of a word $s_{i}$ makes necessary to encode it with a codeword shorter than its current codeword $C_{i}$, a change in the codewords-words mapping is performed. Basically this change simply involves a codewords swapping among words $s_{i}$ and $s_{j}$, where $s_{j}$ has codeword $C_{j}$ and $\left|C_{i}\right|>\left|C_{j}\right|$. As we explain later, $s_{j}$ is the first word (at the top) in the group of words with the same frequency of $s_{i}$. Thus, DLETDC needs two special codewords, $C_{n e w-S y m b o l}$ and $C_{\text {swap. }} C_{\text {new-Symbol }}$ works as was explained for DETDC. $C_{\text {swap }}$ specifies that the receiver should swap the words at the positions pointed by the two codewords that follow $C_{\text {swap }}$. That is, $C_{\text {swap }}, C_{i}, C_{j}$ indicates that now $s_{i}$ is represented by $C_{j}$, and $s_{j}$ by $C_{i}$. This is implemented by a simple swap of words at positions $i$ and $j$ of the vocabulary array in the receiver.

The key idea of this approach is that now there is no correspondence between the word rank (position in the frequencysorted vocabulary of the sender) and its codeword, because words change their positions without changing their codewords. That is, changes in the rank of a word do not produce changes in its codeword except when the codeword must be shorter. Therefore, the sender must maintain an explicit words-codewords mapping, and use it to encode words.

Codewords $C_{n e w-S y m b o l}$ and $C_{\text {swap }}$ can be the first two unused codewords, or the two last codewords of $n$ bytes, being $n$ the codeword size of the last word in the vocabulary in a specific moment of the process. Another possibility is to give them fixed values across the whole process. In our implementation, we used the last two codewords of 3 bytes, since all our experimental corpora can be encoded with codewords of 3 bytes or less. These decisions involve a tradeoff between compression ratio on one hand and decompression and scanning speed on the other, as we will explain later.

Example 1. Figure 1 shows an example of the process carried out by the sender. Assume that, after compressing a portion of a text, word "the" is in the $127^{\text {th }}$ position of the sorted vocabulary, "is" is at the $128^{\text {th }}$ position, and "beautiful" is at the $129^{t h}$ position, all of them with frequency 19.

Assume that the text to be compressed next is "the rose rose beautiful beautiful". After reading "the", the compressor checks that "the" is already in the vocabulary and increases its frequency by 1 . Next, the compressor recomputes the sorted vocabulary. We assume that "the" remains at position 127 . Then the compressor sends codeword $C_{127}$. The next word ("rose") is not in the vocabulary, thus the compressor adds it to the vocabulary at the last position $($ last $=130)$ and gives it the codeword corresponding to that position, $C_{130}$. In order to inform the receiver of this addition, the encoder sends $C_{n e w-S y m b o l}$ and the word "rose" in plain form.

The first occurrence of "beautiful" increases its frequency to 20 and then, after the reorganization, "beautiful" is relocated at the $128^{\text {th }}$ position, swapping it with "is". However, since $C_{128}$ (the codeword representing "is") and $C_{129}$ (the one that represents "beautiful") have the same size (two bytes), the encoder continues using $C_{128}$ to represent "is" and $C_{129}$ to represent "beautiful".

The next occurrence of "beautiful" places it at the $127^{\text {th }}$ position, which has an associated codeword of one byte. Then, since in this case $C_{127}$ and $C_{129}$ have different sizes, the encoder swaps those codewords, associating "the" with $C_{129}$ and "beautiful" with $C_{127}$. Hence, the receiver should be informed of this change. The encoder sends the tuple $C_{\text {swap }}, C_{129}, C_{127}: C_{\text {swap }}$ warns the receiver to expect two codewords that should be exchanged. In addition, the receiver also understands that the second codeword (after the swap) is the word that was actually read. $\square$

The example also illustrates how the position of a word in the sorted vocabulary of the sender is not used to encode a word, thus an explicit mapping must be maintained.

\subsection{Data structures and algorithms}
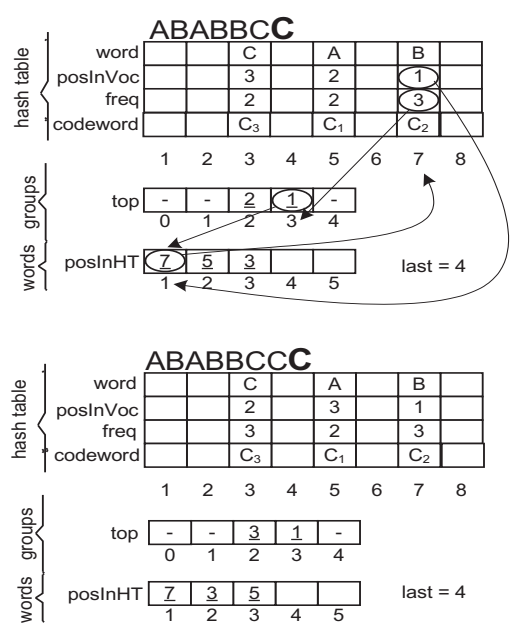

Figure 2: Structures of the sender. 


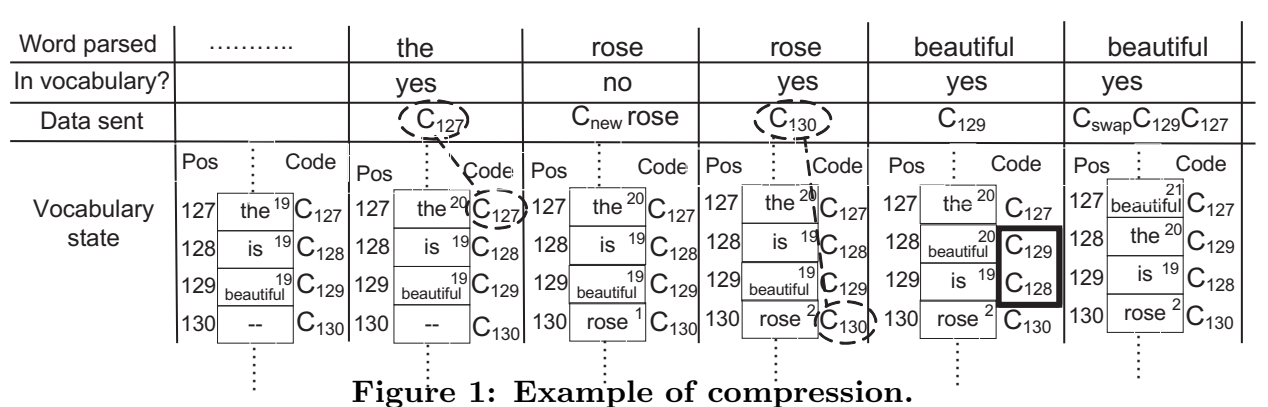

The sender maintains a hash table that permits fast searching for any source word $s_{i}$ to obtain its current position $p$ in the rank, as well as its current frequency and codeword. Essentially, we must be able to identify groups of words with the same frequency, and be able of fast promoting a word to the next group when its frequency increases.

Sender ( )

(1) Initialize vocabulary structures, last $\leftarrow 0$;

(2) for $i \in 1 \ldots m$ do

(3) $\operatorname{read} s_{i}$ from the text;

(4) $\quad p \leftarrow \operatorname{hash}\left(s_{i}\right)$;

(5) if $\operatorname{word}(p)=$ empty (word not in vocabulary) then

(6) send $C_{n e w-S y m b o l}$;

(7) send $s_{i}$ in plain form;

(8) $\quad \operatorname{word}[p] \leftarrow s_{i}$;

(9) $\quad$ posInVoc $[p] \leftarrow$ last;

(10) $\quad$ freq $[p] \leftarrow 1$;

Figure 3: Sender processes in DLETDC

The data structures used by the sender and their functionality are shown in Figure 2. The hash table of words keeps in word the source word, in posInVoc the position of the word in the rank, in freq its frequency and in codeword its codeword. In the vocabulary array (posInHT) words are not explicitly represented, but a pointer to the word slot in the hash table is stored. Finally the array top stores, for each possible frequency, the position in the rank (that is, in array posInHT) of the first word with that frequency. That is, $\operatorname{top}[13]=7$ means that the word in position 7 has frequency 13 , but the word in position 6 has a higher frequency. If no words of frequency $f$ exist, then $t o p[f]=-1$. A variable last storing the first unused position of the vocabulary array is also needed.

When the sender reads a word $s_{i}$, it uses the hash function to obtain its position $p$ in the hash table, so that hash $\left(s_{i}\right)=$ $p$ and therefore word $[p]=s_{i}$. The position of $s_{i}$ in the rank is obtained as $i=\operatorname{posInVoc}[p]$ and its codeword as $C_{i}=\operatorname{codeword}[p]$. In the same way $f=f r e q[p]$. Now,

word $s_{i}$ must be promoted to the next block of frequencies. The sender finds the head of its block as $j=t o p[f]$ and, therefore, the slot of the first word with frequency $f$ in the hash table is $h=\operatorname{posInHT}[j]$ and the codeword of that word is $C_{j}=$ codeword $[h]$.

To promote $s_{i}$ to the next frequency group, it is necessary to swap positions $i$ and $j$ in vector posInHT and in the hash table. The swapping requires exchanging posInHT $[j]=h$ with posInHT $[i]=p$, and exchanging also posInVoc $[p]=$ $i$ and posInVoc $[h]=j$. Once the swapping is done, we promote $j$ to the next block by setting $\operatorname{top}[f]=j+1$. Now we can increase the frequency of $s_{i}, f r e q[p]=f r e q[p]+1$.

If the codeword $C_{j}$ has the same length of $C_{i}$, then $C_{i}$ is sent because it remains as the codeword of $s_{i}$, but if $C_{j}$ is shorter than $C_{i}$ then codeword $[h]=C_{j}$ and codeword $[p]=$ $C_{i}$ are swapped and the sequence $C_{\text {swap }}, C_{i}, C_{j}$ is sent. The receiver will understand that words at positions $i$ and $j$ in its vocabulary array must be swapped and that word $s_{i}$, which from now on will be encoded as $C_{j}$, has been sent.

If $s_{i}$ is a new word, the algorithm sets $\operatorname{word}[p]=s_{i}$, freq $[p]=1, \operatorname{posInVoc}[p]=$ last, codeword $[p]=C_{\text {last }}$ and posInHT $[$ last $]=p$. Then, variable last is incremented and finally codeword $C_{n e w-S y m b o l}$ is sent followed by the word in plain ASCII. Figure 3 shows the pseudocode of the sender.

Receiver ( )

(1) Initialize Vocarray; last $\leftarrow 0$;

(2) for $p \in 1 \ldots m$, do

(3) receive $C_{p}$;

(4) if $C_{p}=C_{n e w-S y m b o l}$ then

(5) receive $s_{p}$ in plain form;

(6) $\quad$ Vocarray [last $] \leftarrow s_{p}$;

(7) $\quad$ last $\leftarrow$ last +1 ;

(8) else

(9) if $C_{p}=C_{\text {swap }}$ then

(10) receive $C_{i}, C_{j}$

(11) $\quad$ swap (Vocarray $\left[\operatorname{decode}\left(C_{i}\right)\right], \operatorname{Vocarray}\left[\operatorname{decode}\left(C_{j}\right)\right]$ ) else output Vocarray $\left[\operatorname{decode}\left(C_{j}\right)\right]$

output Vocarray $\left[\operatorname{decode}\left(C_{p}\right)\right]$;

Figure 5: Receiver processes in DLETDC

In the receiver, a simple words array Vocarray and a variable last are the only necessary structures, as explained. Words are introduced in the vocabulary array as they arrive, always at the last position. Therefore, there is a implicit mapping among word position and codewords, as in ETDC. This fact permits using the same decode procedure used for ETDC. The difference is that, in DLETDC, the receiver does not take account of the frequency of the words and does not update their position in the vocabulary accordingly to their frequency. Words in Vocarray are, in fact, sorted by codeword, and two words are swaped, always following sender 


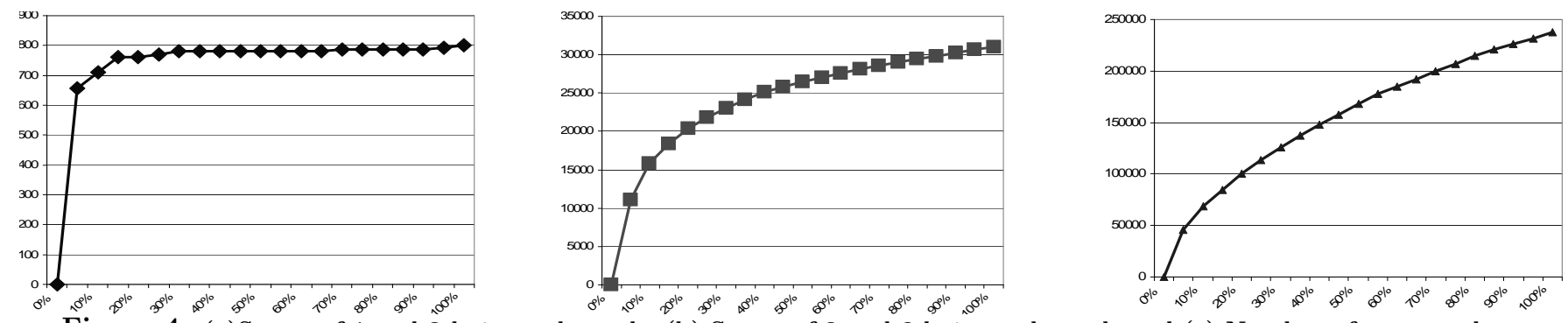

Figure 4: (a)Swaps of 1 and 2 bytes codewords, (b) Swaps of 2 and 3 bytes codewords and (c) Number of new words.

instructions, when an exchange in their codeword lengths is needed to keep the original DETDC compression ratio. Figure 5 shows the pseudocode for the receiver process. Observe that the receiver only has to follow the instructions of the sender, that is, insert new symbols in the words vector (sorted by codewords) when $C_{n e w-s y m b o l}$ arrives, and swap two words in the vector when $C_{\text {swap }}$ arrives.

\section{EXPERIMENTAL RESULTS}

We used some large text collections from TREC-2, namely AP Newswire 1988 (AP) and Ziff Data 1989-1990 (ZIFF), as well as TREC-4, namely Congressional Record 1993 (CR) and Financial Times 1991 to 1994 (FT91 to FT94). We created two larger corpora ALLFT and ALL by aggregating all texts from FT and of all corpora respectively. As a small collection we use the Calgary corpus. We used the spaceless word model [9] to create the vocabulary, that is, if a word was followed by a space, we just encoded the word, otherwise both the word and the separator were encoded.

A dual Intel ${ }^{\circledR}$ Pentium ${ }^{\circledR}$-III $800 \mathrm{Mhz}$ system, with 768 MB SDRAM-100Mhz was used in our tests. It ran Debian GNU/Linux (kernel version 2.2.19). The compiler used was gcc version 3.3.3 20040429 and -09 compiler optimizations were used. Time results measure CPU user time in seconds.

\subsection{Swaps and evolution of new words}

Remember that using bytes as target symbols and an ETDC approach, words from position 0 to position 127 are encoded with one byte, words in positions form 128 to $128+128^{2}$ are encoded with two bytes and so on. With 3 bytes, it is possible to encode more than two millions of words (and vocabularies very rarely will be so huge given Heaps law [12]). Therefore there are two very clear limits in the sorted vocabulary, 128 and $128+128^{2}$. These two limits set three intervals. Only when a word changes its position in a way that it changes its ranking interval, the corresponding codeword is modified. Therefore, and this is the key idea, very few changes will occur in the mapping codewords-words. That is, very few swaps will be needed.

We compressed collection ZIFF to show how the number of swaps evolves as the text is compressed. The file has 40,627,132 words, and a vocabulary of 237,622 (different) words. In the compression process, 31,772 swaps were produced. This means that only the $0.078 \%$ of the frequency changes implied a swap. In addition, most of these swaps were produced in the first stages of the compression, as it can be seen in Figure 4 .

Note that most of the changes $(30,971)$ are between codewords of size 2 and 3 . This is expected since the shape of the Zipf distribution signals a bigger difference in frequency between words $127^{\text {th }}$ and $128^{\text {th }}$ than between words in po- sitions $128+128^{2}-1$ and $128+128^{2}$. Notice that if the word in a position $128+128^{2}-1$ has frequency 1 , just one occurrence of any word at position lower than $128+128^{2}$ will produce a swap.

Observe in Figure 4(a) that most of the swaps involving codewords of 1 and 2 bytes take place during the processing of the first $5 \%$ of the file. After processing $20 \%$ of the file, there are almost no further swaps of this type.

Many (about 30\%) of the swaps between codewords of 2 and 3 bytes also take place during the compression of the first $5 \%$ of the file (see Figure 4(b)). The number of swaps decreases as the compression progresses, showing that the model gets closer to the real distribution as the sample grows. However, the convergence is slower. Figure4(c)) shows that the distribution of new words, as expected, follows a Heaps' law.

\subsection{Compression ratio and efficiency}

Our implementation of DLETDC uses fixed three-byte values for $C_{n e w-S y m b o l}$ and $C_{\text {swap }}$, producing a small lose in compression. If we uses the first two free codewords, we would have saved 2 bytes for $C_{n e w-S y m b o l}$ in the 127 first new words and one byte in the $128^{2}=16,384$ next words. That is, we would have saved 16,640 bytes for codeword $C_{n e w-S y m b o l}$. The same reasoning can be done for $C_{\text {swap }}$. We have lost exactly 534 bytes by using a fixed three-byte $C_{\text {swap }}$ in the compression of the ZIFF collection shown in Figure 4. These 17, 174 bytes are insignificant in the compressed text size. Table 1 shows the compression ratio we obtained in the different corpora using the two alternatives. Column "Variable" shows the compression ratio reached using the first two free codewords, while "Fixed" refers to using the last two codewords of three bytes. Notice that the loss in compression ratio is insignificant, especially on large files.

\begin{tabular}{|l|r|r|r|}
\hline Corpus & \multicolumn{1}{|c|}{ Size } & Variable & Fixed \\
\hline CALGARY & $2,131,045$ & $49.36 \%$ & $50.16 \%$ \\
FT91 & $14,749,355$ & $36.16 \%$ & $36.28 \%$ \\
CR & $51,085,545$ & $32.21 \%$ & $32.24 \%$ \\
FT92 & $175,449,235$ & $32.93 \%$ & $32.94 \%$ \\
ZIFF & $185,220,215$ & $33.87 \%$ & $33.88 \%$ \\
FT93 & $197,586,294$ & $32.97 \%$ & $32.98 \%$ \\
FT94 & $203,783,923$ & $32.92 \%$ & $32.93 \%$ \\
AP & $250,714,271$ & $33.17 \%$ & $33.18 \%$ \\
FTALL & $591,568,807$ & $32.57 \%$ & $32.57 \%$ \\
ALL & $1,080,719,883$ & $33.69 \%$ & $33.69 \%$ \\
\hline
\end{tabular}

Table 1: Comparing compression ratios.

The computationally most significant work at the receiver is to process the swap, and as we have shown, the number of swaps occurred is insignificant with respect to the number of words processed. In addition, the number of swaps drops as 


\begin{tabular}{|l|r|r|r|r|r|}
\hline Corpus & Gzip & DETDC & DETDC & Arith & Bzip2 \\
\hline CALG. & $36,95 \%$ & $50,16 \%$ & $47,73 \%$ & $34,68 \%$ & $28,92 \%$ \\
FT91 & $36,42 \%$ & $36,28 \%$ & $35,64 \%$ & $28,33 \%$ & $27,06 \%$ \\
CR & $33,29 \%$ & $32,24 \%$ & $31,98 \%$ & $26,30 \%$ & $24,14 \%$ \\
FT92 & $36,47 \%$ & $32,94 \%$ & $32,84 \%$ & $29,82 \%$ & $27,09 \%$ \\
ZIFF & $33,06 \%$ & $33,88 \%$ & $33,79 \%$ & $26,36 \%$ & $25,11 \%$ \\
FT93 & $34,21 \%$ & $32,98 \%$ & $32,89 \%$ & $27,89 \%$ & $25,32 \%$ \\
FT94 & $34,21 \%$ & $32,93 \%$ & $32,84 \%$ & $27,86 \%$ & $25,26 \%$ \\
AP & $37,31 \%$ & $33,18 \%$ & $33,11 \%$ & $28,00 \%$ & $27,21 \%$ \\
FTALL & $34,94 \%$ & $32,57 \%$ & $32,54 \%$ & $27,85 \%$ & $25,86 \%$ \\
ALL & $35,09 \%$ & $33,69 \%$ & $33,66 \%$ & $27,98 \%$ & $25,98 \%$ \\
\hline
\end{tabular}

Table 2: Compression Ratio.

\begin{tabular}{|l|r|r|r|r|r|}
\hline Corpus & Gzip & DLETDC & DETDC & Arith & Bzip2 \\
\hline CALG. & 0.933 & 0.470 & 0.384 & 1.030 & 2.660 \\
FT91 & 6.303 & 2.990 & 2.488 & 6.347 & 18.200 \\
CR & 19.767 & 10.010 & 8.418 & 21.930 & 65.170 \\
FT92 & 76.313 & 36.983 & 31.440 & 80.390 & 221.460 \\
ZIFF & 71.293 & 39.370 & 33.394 & 82.720 & 233.250 \\
FT93 & 77.560 & 41.823 & 36.306 & 91.057 & 237.750 \\
FT94 & 73.290 & 43.703 & 36.718 & 93.467 & 255.220 \\
AP & 114.570 & 54.647 & 47.048 & 116.983 & 310.620 \\
FTALL & 234.703 & 127.745 & 111.068 & 274.310 & 718.250 \\
ALL & 431.470 & 254.910 & 213.905 & 509.710 & 1.342 .430 \\
\hline
\end{tabular}

Table 3: Compression time.

the model converges, and therefore, since the receiver does very little computation, it is very fast.

We empirically compare the compression ratio and compression/decompression speed of DLETDC, against Gzip (a Ziv-Lempel compressor) [23, 24], Bzip2 (a block sorting compressor) [7], an arithmetic encoder [8] and DETDC [5].

Table 2 shows the results on compression ratio. As expected Bzip2 compresses the most and Gzip the least. DLETDC achieves better compression ratios than Gzip except in small collections (Calgary). DLETDC compresses slightly less than DETDC, since the compressed data has to carry information about the swaps.

Table 3 shows compression time. Now it is clear that DETDC and DLETDC are by far the best anternatives. Notice that due to the swap management, DLETDC is slightly worse than the DETDC. Table 4 shows decompression times. Remember that Gzip is considered a very efficient technique for decoding, and in fact this is its strength when compared to DETDC and the others. However, DLETDC is never much slower than Gzip and it is clearly faster than Gzip as soon as the collection has a medium size.

Hence, DLETDC is easier to program, compresses more, and compresses and decompresses faster Gzip. This is enough by itself to make DLETDC an interesting choice for dynamic compression of natural language texts. However, it has other relevant features, as we show next.

\section{KEYWORD FILTERING}

\begin{tabular}{|l|r|r|r|r|r|}
\hline & Gzip & DLETDC & DETDC & Arith & Bzip2 \\
\hline CALGARY & 0.067 & 0.097 & 0.240 & 0.973 & 0.830 \\
FT91 & 0.673 & 0.600 & 1.545 & 5.527 & 5.890 \\
CR & 2.123 & 2.013 & 5.265 & 18.053 & 19.890 \\
FT92 & 7.703 & 7.850 & 19.415 & 65.680 & 71.050 \\
ZIFF & 7.577 & 7.947 & 20.690 & 67.120 & 72.340 \\
FT93 & 9.120 & 8.610 & 21.935 & 71.233 & 77.860 \\
FT94 & 9.160 & 8.933 & 22.213 & 75.925 & 80.370 \\
AP & 13.057 & 11.650 & 27.233 & 88.823 & 103.010 \\
FTALL & 28.823 & 25.735 & 66.238 & 214.180 & 235.370 \\
ALL & 59.790 & 49.530 & 126.938 & 394.067 & 432.390 \\
\hline
\end{tabular}

Table 4: Decompression time.
The problem to perform direct search over text compressed with previous dynamic methods is that the codeword, used to encode a specific word, changes many times along the process. Following those changes requires an effort close to that of just decompressing the text and then searching it.

Since, as we showed, there are very few swaps, the codeword of each word changes with much less frequency than in the case of previous adaptive techniques. This makes it possible to scan the arriving text looking for some specific patterns. Of course the codewords for those patterns may change along the process. At the beginning of the process, a searched word will appear in ASCII form, preceded by the $C_{n e w-S y m b o l}$ codeword. At that point the $C_{\text {last }}$ codeword becomes the pattern we must look for to find that word. Latter, that codeword may change again but the $C_{\text {swap }}$ codeword marks each change and, therefore, the scanning algorithm can easily follow the evolution of all the search patterns in the compressed text.

Since we have few changes in the codeword-word mapping as we show in Section 4.1, we can apply a Boyer-Moore family search algorithm, which in our experiments has been the set Horspool algorithm [13, 18]. However, we have to consider several special issues when searching DLETDC compressed text. Let us suppose that we are searching for patterns $p_{1}, p_{2}, \ldots, p_{n}$. We use $P\left(p_{i}\right), 1 \leq 1 \leq n$ to denote the plain version of the pattern and $C\left(p_{i}\right)$ to denote the codeword representing $p_{i}$ in a certain moment.

We represented the patterns, both ASCII versions of the words and codewords, using a trie. Initially all the $P\left(p_{i}\right)$ are represented in the trie, but as soon as any $P\left(p_{i}\right)$ appears for the first time in the text, the trie is updated by deleting $P\left(p_{i}\right)$ and inserting the corresponding $C\left(p_{i}\right)$ pattern (which initially is always the current $C_{\text {last }}$ value). Codeword $C_{\text {swap }}$ is also represented in the trie, since the codeword of a search pattern can be changed by the sender using $C_{\text {swap }}$ as the escape codeword. Upon finding $C_{\text {swap }}$, the algorithm has to read the next two codewords and check if one (or both) of them is in the trie. If it is, the algorithm updates the trie in order to replace the current $C\left(p_{i}\right)$ codeword of a search pattern by its new codeword.

Remember that, although we lose some compression, we preferred to use for $C_{n e w-S y m b o l}$ and $C_{\text {swap }}$ the last threebyte codewords, because this improves the search speed. Both $C_{n e w-S y m b o l}$ and $C_{\text {swap }}$ must be explicitly represented in the trie, so if we used the first two free codewords to represent them, we would have to update the trie each time a new word arrived, which would be too expensive.

On the other hand, a Booyer-Moore pattern matching algorithm takes advantage of longer patterns because it permits skipping more bytes. The use of variable values for $C_{n e w-S y m b o l}$ and $C_{\text {swap }}$ would yield too short codewords to look for at the beginning of the process. The fixed alternative of three-byte codewords permits longer shifts (unless we search for a frequent word coded with one or two bytes).

\subsection{Empirical results on searching}

We searched, in both the compressed and the uncompressed versions of the text, the same set of patterns chosen at random from the text. We averaged the results over 100 searches, each with different patterns. Patterns that occurred only once in the test were not chosen, to avoid strange elements such as long arrays of separator characters.

Table 5 compares search times over corpora of different 


\begin{tabular}{|l|llll|ll|ll|l|l|}
\hline Patterns & \multicolumn{3}{|c|}{ CR } & \multicolumn{3}{|c|}{ AP } & \multicolumn{3}{c|}{ ALL } \\
\hline$\#$ & len. & PlainDLETDC (\%) & PlainDLETDC (\%) & \multicolumn{3}{|c|}{ Plain DLETDC (\%) } \\
\hline 5 & 6 & 0.47 & 0.20 & 42.55 & 2.40 & 0.92 & 38.33 & 10.14 & 3.52 & 34.74 \\
5 & 1 & 0.51 & 0.20 & 38.83 & 2.62 & 0.92 & 35.30 & 11.76 & 3.36 & 28.61 \\
\hline 10 & 6 & 0.62 & 0.21 & 33.87 & 3.14 & 0.98 & 31.32 & 13.80 & 3.46 & 25.06 \\
10 & 1 & 0.73 & 0.21 & 28.76 & 3.60 & 0.97 & 26.90 & 17.12 & 4.17 & 24.35 \\
\hline 15 & 6 & 0.73 & 0.21 & 28.76 & 3.68 & 1.02 & 27.71 & 15.84 & 4.38 & 27.64 \\
15 & 1 & 0.89 & 0.22 & 24.71 & 4.31 & 1.01 & 23.40 & 19.18 & 4.33 & 22.57 \\
\hline 20 & 6 & 0.80 & 0.22 & 27.50 & 4.09 & 1.06 & 25.91 & 17.61 & 4.49 & 25.49 \\
20 & 1 & 0.99 & 0.23 & 23.23 & 5.18 & 1.06 & 20.56 & 20.47 & 4.49 & 21.93 \\
\hline 25 & 6 & 0.88 & 0.35 & 39.77 & 4.35 & 1.26 & 29.08 & 18.70 & 5.01 & 26.81 \\
25 & 1 & 1.12 & 0.23 & 20.53 & 5.57 & 1.21 & 21.72 & 23.14 & 4.63 & 20.03 \\
\hline
\end{tabular}

Table 5: Searching in CR, AP and ALL corpora.

sizes. The first column gives the number and minimum ASCII length of the search patterns. For each corpus we give the time to search the plain and the uncompressed text, as well as the compressed/uncompressed time fraction.

The use of longer patterns obviously improves the search speed in the uncompressed text. However, this has little effect in the search time over the compressed text, as in this case we always search for codes of two or three bytes (rarely shorter, as only 128 words are encoded with just one byte).

A more surprising result is that a larger number of patterns favors the search of the compressed over the uncompressed text. Note that handling more patterns does not affect much the search speed in DLETDC compressed text. The main reason is that Horspool algorithm benefits from a lower probability of two characters (from the text and the pattern) being equal. The lower this probability, the more patterns can be handled efficiently. In DLETDC compressed text this probability is close to $1 / 256 \approx 0.004$, while in plain English text it is known to be around 0.067 .

\section{CONCLUSIONS}

We have presented Dynamic Lightweight End Tagged Dense Code (DLETDC), a new word-based byte oriented statistical compressor that, as ETDC [6], (sc)-DC [4] and DETDC [5], belongs to the family of dense compressors. DLETDC, as other statistical word-oriented compressors, obtains compression ratios around $32-34 \%$ but, being a member of the "dense" family, it is easier to implement and, at the same time, compresses more efficiently.

We show that, as soon as the compressed text collection reaches a significative size, DLETDC becomes an interesting alternative because it is more efficient and obtains better compression ratios than DETDC and Gzip. At the same time DLETDC offers an interesting space/time tradeoff that no other dynamic compressor, such as bzip2 or arithmetic encoders, can reach. A very interesting feature of this compressor is that it breaks the usual symmetry between sender and receiver processes, which is common to most adaptive compressors. Two interesting consequences of this asymmetry are: the low computing power required by the receiver, where decompression is remarkably fast, and the possibility of searching the compressed text without decompressing it, uncommon on adaptive scenarios.

This second property opens the possibility of scanning the compressed text as it arrives and filtering it by the words it contains to perform classification or retrieval tasks. The possibility of scanning a dynamic compressed text as it is arriving opens a new horizon of possibilities for new applications and environments, such as mobile computing when a source continuously broadcasts information.

\section{REFERENCES}

[1] R. Baeza-Yates and B. Ribeiro-Neto. Modern Information Retrieval. Addison-Wesley, 1999.

[2] T. C. Bell, J. G. Cleary, and I. H. Witten. Text Compression. Prentice Hall, 1990.

[3] R. Boyer and J. Moore. A fast string searching algorithm. Comm. ACM, 20(10):762-772, 1977.

[4] N. Brisaboa, A. Fariña, G. Navarro, and M. Esteller. (s,c)-dense coding: An optimized compression code for natural language text databases. In Proc. $10^{\text {th }}$ SPIRE, LNCS 2857, pages 122-136, 2003.

[5] N. Brisaboa, A. Fariña, G. Navarro, and J. Paramá. Simple, fast, and efficient natural language adaptive compression. In Proc. $11^{\text {th }}$ SPIRE, LNCS 3246, pages 230-241, 2004.

[6] N. Brisaboa, E. Iglesias, G. Navarro, and J. Paramá. An efficient compression code for text databases. In Proc $25^{\text {th }}$ ECIR, LNCS 2633, pages 468-481, 2003.

[7] M. Burrows and D. J. Wheeler. A block-sorting lossless data compression algorithm. Tech.Rep. 124, Digital, 1994.

[8] J. Carpinelli, A. Moffat, R. Neal, W. Salamonsen, L. Stuiver, A. Turpin, and I. Witten. Word, character, integer, and bit based compression using arithmetic coding. http://www.cs.mu.oz.au/ alistair/arith_coder/, 1999.

[9] E. de Moura, G. Navarro, N. Ziviani, and R. Baeza-Yates. Fast searching on compressed text allowing errors. In Proc. $21^{\text {st }}$ SIGIR, pages 298-306, 1998.

[10] E. de Moura, G. Navarro, N. Ziviani, and R. Baeza-Yates. Fast and flexible word searching on compressed text. $A C M$ TOIS, 18(2):113-139, 2000.

[11] A. Fariña. New Compression Codes for Text Databases. $\mathrm{PhD}$ thesis, University of A Coruña, Computer Science Department, A Coruña, Spain, 2005. To appear.

[12] H. S. Heaps. Information Retrieval: Computational and Theoretical Aspects. Academic Press, 1978.

[13] R. N. Horspool. Practical fast searching in strings. Software Practice and Experience, 10(6):501-506, 1980.

[14] D. A. Huffman. A method for the construction of minimum-redundancy codes. Proc. Inst. Radio Eng., 40(9):1098-1101, 1952.

[15] A. Moffat. Word-based text compression. Software Practice and Experience, 19(2):185-198, 1989.

[16] A. Moffat and A. Turpin. Compression and Coding Algorithms. Kluwer, 2002.

[17] G. Navarro, E. de Moura, M. Neubert, N. Ziviani, and R. Baeza-Yates. Adding compression to block addressing inverted indexes. Information Retrieval, 3(1):49-77, 2000.

[18] G. Navarro and M. Raffinot. Flexible Pattern Matching in Strings - Practical on-line search algorithms for texts and biological sequences. Cambridge University Press, 2002.

[19] G. Navarro and J. Tarhio. Boyer-Moore string matching over Ziv-Lempel compressed text. In Proc. $11^{\text {th }} C P M$, LNCS 1848, pages 166-180, 2000.

[20] A. Turpin and A. Moffat. Fast file search using text compression. In Proc. 20 $0^{\text {th }}$ Australian Computer Science Conf., pages 1-8, 1997.

[21] I. H. Witten, A. Moffat, and T. C. Bell. Managing Gigabytes: Compressing and Indexing Documents and Images. Morgan, 1999.

[22] G.K. Zipf. Human Behavior and the Principle of Least Effort. Addison-Wesley, 1949.

[23] J. Ziv and A. Lempel. A universal algorithm for sequential data compression. IEEE TIT, 23(3):337-343, 1977.

[24] J. Ziv and A. Lempel. Compression of individual sequences via variable-rate coding. IEEE TIT, 24(5):530-536, 1978.

[25] N. Ziviani, E. de Moura, G. Navarro, and R. Baeza-Yates. Compression: A key for next-generation text retrieval systems. Computer, 33(11):37-44, 2000. 